\title{
Relationship of Naturally Occurring Antisperm Antibodies in Blood Serum and Seminal Plasma of Cattle Bulls with Sperm Function and Fertility Tests
}

\author{
V. Zodinsanga ${ }^{1}$, Ranjna S. Cheema ${ }^{1}$, P. S. Mavi² \\ ${ }^{1}$ Department of Veterinary Gynaecology and Obstetrics, Guru Angad Dev Veterinary and Animal Sciences \\ University, Ludhiana, India \\ ${ }^{2}$ Department of Teaching Veterinary Clinical Complex, Guru Angad Dev Veterinary and Animal Sciences \\ University, Ludhiana, India \\ Email: ${ }^{*}$ Ranjna.cheema@gmail.com
}

Received 12 February 2015; accepted 28 March 2015; published 1 April 2015

Copyright (C) 2015 by authors and Scientific Research Publishing Inc.

This work is licensed under the Creative Commons Attribution International License (CC BY). http://creativecommons.org/licenses/by/4.0/

(c) (i) Open Access

\section{Abstract}

The study was planned with an objective to assess the level of antisperm antibodies (ASA) in the blood serum and seminal plasma of breeding cow bulls and their relationship with sperm function and fertility tests. ASA was analyzed in blood serum and seminal plasma by SpermMar test, Immuno peroxidase assay (IPA) and Enzyme linked immunoabsorbant assay (ELISA). In SpermMar test, about $54 \%$ bulls were with $\mathbf{7 0} \%$ IgG in blood serum against sperm surface antigens, whereas none of the bulls were with $>10 \%$ IgG in seminal plasma. More than $20 \%$ and $>10 \%$ IgA against sperm surface antigens were detected in the blood serum and seminal plasma of $65.8 \%$ and $37 \%$ bulls, respectively. Out of 26 bulls, seminal plasma of 21 bulls reacted with spermatozoa both in IPA and IgA latex particles and that of only 12 bulls reacted with IgG. In IPA, about $50 \%$ of the bulls had $>40 \%$ ASA against head surface antigens, whereas, there were $23 \%$ bulls with $>10 \%$ ASA in seminal plasma. Also ELISA indicated a higher antibody titre in blood serum $(3200-6400)$ and seminal plasma ( $40-80$ ) of $50 \%$ and $42 \%$ bulls, respectively. There were 11 bulls with low values of HOST/in vitro acrosome reaction/cervical mucus penetration assay and higher level of either serum or seminal plasma ASA. Our study revealed that a significant level of ASA in serum or seminal plasma may have effect on the fertility of bulls by affecting the sperm function.

\section{Keywords}

ASA, Cattle Bulls, Sperm-Function, Fertility-Tests, Relationship

\footnotetext{
${ }^{*}$ Corresponding author.
}

How to cite this paper: Zodinsanga, V., Cheema, R.S. and Mavi, P.S. (2015) Relationship of Naturally Occurring Antisperm Antibodies in Blood Serum and Seminal Plasma of Cattle Bulls with Sperm Function and Fertility Tests. Open Journal of Animal Sciences, 5, 114-123. http://dx.doi.org/10.4236/ojas.2015.52014 


\section{Introduction}

Sperm has long been known to be antigenic and antisperm antibodies (ASA) are immune-reactive proteins produced by the body in response to the sperm antigens. The blood-testis barrier (BTB), a tight junction among sertoli cells, separates the spermatozoa and immune system. It prevents testicular cells, which express foreign antigens from coming into contact with lymphoid tissue and immunocompetant cells. However, BTB is commonly disrupted by physiological leakage of normally sequestered sperm antigens. Generally, ASA formation can be induced primarily during infectious and non-infectious inflammations, or by obstruction of testicular efferent duct [1]. Sperm antibodies may be both transudates from the blood and secreted locally by plasma cells within the reproductive tract [2]. Sperm-reactive antibodies can also be present in serum yet undetectable in semen or within female reproductive tract secretions [3]. ASA has harmful effects on sperm function and may prevent motility or lead to sperm death. Negative effect of ASA on sperm function during in vitro fertilization (IVF) was demonstrated by Kim et al. and Lombardo et al. [4] [5]. An elaborative study done by Zraly et al. [1] indicated that ASA was significantly higher in active donors of semen compared with the candidate breeders that had not been used as regular donors $(56.4 \%$ vs. $39.2 \%$; $\mathrm{P}<0.01)$ and fertility in those bulls was insignificantly lower. The study was done with an objective to evaluate the relationship of ASA with sperm function and fertility tests in cattle bulls.

\section{Material and Methods}

\subsection{Procurement of Samples}

Frozen semen of 26 breeding cattle bulls (Pure HF and HF crosses) were procured from Semen Freezing Laboratory, GADVASU, Ludhiana, Semen Bank Bhattian, Khanna and Government Semen Bank, Ropar, Punjab, India during 2013-2014. Freshly ejaculated semen and blood of these bulls were also collected to harvest seminal plasma and serum, respectively from the respective farms. All the tests were performed in duplicate.

\subsection{Semen Function and Fertility Tests}

\subsubsection{Sperm Concentration}

Sperm concentration was taken from the records of respective semen freezing laboratories.

\subsubsection{Individual Motility}

A drop of frozen-thawed semen was placed on micro slide, covered with cover slip and progressively motile spermatozoa were observed under bright field microscope $(400 \times)$ at $37^{\circ} \mathrm{C}$. A total of 200 spermatozoa were counted in different fields and percentage motility was calculated.

\subsubsection{Sperm Viability and Abnormalities [6]}

Frozen thawed semen was evaluated for viability and abnormalities through Eosin Nigrosin staining method. A total of 150 sperms were counted in different fields under oil immersion at $1000 \mathrm{X}$ and per cent live sperm was calculated. Spermatozoa with various abnormalities of head, tail and mid piece were also observed in eosin-nigrosin stained slides.

\subsubsection{Hypo Osmotic Swelling Test [7]}

A total of 150 spermatoazoa were counted at 400X in different fields and total number of coiled tailed sperms was calculated. The number of coiled tailed spermatozoa in phosphate buffered saline (PBS) was deducted from the number in hypo osmotic solution and the resultant figure was taken as the HOS reactive spermatozoa.

\subsubsection{Cervical Mucus Penetration Test [8]}

Cervical mucus was collected from a normal cycling cow in estrus and was filled in a capillary by capillary action. Capillary was sealed from one side with PVA powder and pre heated at $37^{\circ} \mathrm{C}$ for 10 min. Approximately $100 \mu \mathrm{l}$ of frozen semen was placed at the bottom of an eppendorf tube and a capillary tube was placed with its open end in the semen. After $30 \mathrm{~min}$ of incubation at $37^{\circ} \mathrm{C}$, the capillary tube was fixed on scaled glass slide and viewed under microscope at $400 \times$. The length of the tube was then scanned to establish the distance furthest from the semen reservoir attained by spermatozoa. The maximum distance of migration of spermatozoa after 30 
min of incubation was defined as the migration distance. Number of migrated spermatozoa was counted in the peak $0.5 \mathrm{~cm}$.

\subsubsection{Acrosomal Integrity [9]}

Sperm smears stained with giemesa were examined microscopically under oil immersion at $1000 \mathrm{X}$. About 200 spermatozoa with intact acrosomes (stained dark purple) and damaged acrosome (without stain/stained light purple) was counted in different fields and percentage of spermatozoa with intact acrosomes was calculated.

\subsubsection{In Vitro Capacitation and Acrosome Reaction [10]}

About $200 \times 10^{6}$ spermatozoa/ml were incubated in Tyrode albumin lactate pyruvate (TALP) medium (100 mM $\mathrm{NaCL}, 3.1 \mathrm{mM} \mathrm{KCl}, 25 \mathrm{mM} \mathrm{NaHCO}_{3}, 0.3 \mathrm{mM} \mathrm{Na}_{2} \mathrm{HPO}_{4}, 21.6 \mathrm{mM}$ Na lactate, $2 \mathrm{mM} \mathrm{CaCl}, 0.4 \mathrm{mM} \mathrm{MgCl}$ $4 \mathrm{H}_{2} \mathrm{O}, 10 \mathrm{mM}$ Hepes, $1 \mathrm{mM}$ Na pyruvate, $0.6 \%$ bovine serum albumin (BSA), $5 \mathrm{mM}$ glucose and heparin 10 $\mu \mathrm{g} / \mathrm{ml}$ ) at $37^{\circ} \mathrm{C}$ in an incubator for $4-6 \mathrm{hrs}$. Motility was checked every hour and sperm smears were prepared at 0,4 and 6 hrs of incubation. Sperm smears were stained with giemsa to observe different stages of acrosome reaction. Giemsa stained slides were observed under bright field microscope at 1000X and about 200 spermatozoa with swollen heads, vesiculated and shedded acrosomes were counted.

\subsubsection{Calculation of Percentage of Spermatozoa}

Percentage of motile, viable, HOS reactive and acrosome reacted spermatozoa was calculated by the following formula:

$$
\frac{\text { Number of motile/viable/HOS reactive/acrosome reacted spermatozoa }}{\text { Total counted spermatozoa }} \times 100
$$

Total counted spermatozoa

\subsection{Detection of ASA in Serum and Seminal Plasma}

\subsubsection{Preparation of Blood Serum and Seminal Plasma}

Blood serum and seminal plasma were obtained by centrifugation of clotted blood and fresh semen at $3000 \mathrm{rpm}$ for $10 \mathrm{~min}$, respectively. Blood serum and seminal plasma were heated at $56^{\circ} \mathrm{C}$ for $30 \mathrm{~min}$ to inactivate complements.

\subsubsection{Immunoperoxidase Assay (IPA) [11]}

Sperm smears on clean slides were incubated with $1 \%$ bovine sperm albumin for 2 hours at $4^{\circ} \mathrm{C}$. Slides were washed thrice with PBS, $\mathrm{pH} 7.4$, incubated with 1:200 diluted serum/1:10 diluted seminal plasma for 1 hour at $37^{\circ} \mathrm{C}$, again washed thrice with PBS. Smears were incubated with rabbit anti-bovine IgG (Sigma) for 45 minutes at $37^{\circ} \mathrm{C}$ and washed thrice with PBS. Colour was developed with 3, 3'-Diaminobenzindine tetrahydrochloride in Tris buffer $\left(0.05 \mathrm{M}, \mathrm{pH} 7.6\right.$ at $\left.25^{\circ} \mathrm{C}\right)$ and $27 \mu \mathrm{l}$ of $3 \%$ hydrogen peroxide for 5 minutes at room temperature. Washed with distilled water, slides were mounted in $10 \%$ glycerol in PBS, covered with coverslip and examined under the microscope at $10 \times 100 \mathrm{X}$ for dark brownish colouration of the sperm. About 200 sperms with browning on acrosome, post acrosomal cap or whole head were counted in different fields and percentage of IPA positive sperms were calculated (Figure 1).

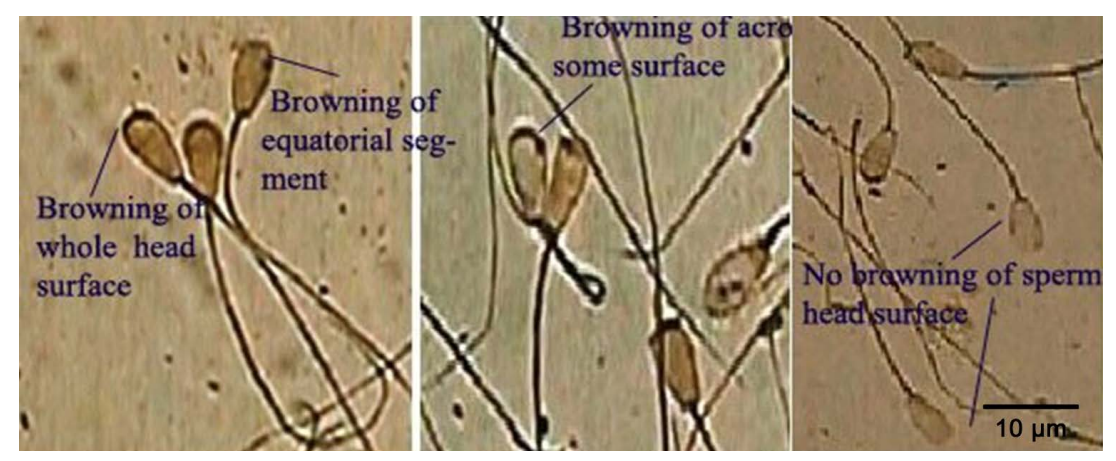

Figure 1. Showing browning of sperm membrane in immunoperoxidase assay. 


\subsubsection{Indirect Sperm Mar Test with Sperm Mar Kit [12]}

Diluted inactivated serum/cervical mucus $1 / 4$ with TALP medium, $\mathrm{pH}, 7.4$ and incubated at $37^{\circ} \mathrm{C}$ for $30 \mathrm{~min}$. Collected the motile sperms by centrifugation through Histopaque, suspended the sperm pellet in TALP and adjusted the sperm conc to $20 \times 10^{6}$. Incubated $100 \mu 1$ of the sperm suspension of motile spermatozoa with $100 \mu 1$ of inactivated $1 / 4$ diluted serum or seminal plasma, incubated for 1 hour at $37^{\circ} \mathrm{C}$. Added $2 \mathrm{ml}$ of TALP, mixed well and centrifuged for 10 minutes at $400 \mathrm{~g}$. Resuspended the pellet with $50 \mu 1$ of TALP. On a slide, mixed 10 $\mu \mathrm{l}$ of sperm suspension and $5 \mu \mathrm{l}$ of sperm Mar latex particles bound to $\operatorname{IgG} / \operatorname{IgA}$, covered with cover slip, kept in humid chamber for $5 \mathrm{~min}$ and observed under microscope at $400 \mathrm{X}$. Attachment of latex particles to the head/tail or whole sperm was observed (Figure 2). About 200 sperms in different fields were counted and percentage was calculated.

\subsubsection{Enzyme Linked Immunoabsorbant Assay (ELISA) [13]}

Sperm antigen/extracts were prepared by suspending washed spermatozoa in $62.5 \mathrm{mM}$ Tris-HCl, $\mathrm{pH} 6.8$ containing 2\% SDS, $1 \mathrm{mM}$ PMSF, $25 \mathrm{mM}$ benzidine, $10 \mathrm{mM}$ aprotinin, $10 \mathrm{mM}$ pepstatin and $5 \mathrm{mM}$ soyabean trypsin inhibitor, sonicated ( 3 bursts of $20 \mathrm{sec}$ each) and centrifuged at 15,000 rpm for 30 minutes. ELISA plates were coated with $5 \mu \mathrm{g}$ protein (sperm antigen) per well by incubating at $37^{\circ} \mathrm{C}$ for three hrs. After washing thrice with PBS, antigen coating was blocked by incubating with $300 \mu 1$ of $2 \%$ BSA per well for overnight at $4^{\circ} \mathrm{C}$. Again washed thrice with PBS pH 7.4 and added serial dilutions of serum/cervical mucus into the wells and incubated at $37^{\circ} \mathrm{C}$ for three hours. Washed again with PBS and incubated with $100 \mu 1 /$ well of HRP conjugated anti bovine IgG for three hours at $37^{\circ} \mathrm{C}$. Washed the plate twice with PBS and incubated with $100 \mu \mathrm{l}$ of o-phenyldiamine $+0.06 \% \mathrm{H}_{2} \mathrm{O}_{2}$ as a substrate for $20 \mathrm{~min}$ at room temperature. Stopped the reaction with $5 \mathrm{~N}_{2} \mathrm{SO}_{4}$ and measured the absorbance at $492 \mathrm{~nm}$ using ELISA reader.

\subsubsection{Statistical Analysis}

The data obtained was analyzed statistically according to Independent Sample T-Test and One-Way ANOVA using difference between means of two groups and means of different group application at 5 per cent level of significance (SPSS, Version 16.0).

\section{Results}

\subsection{Immunoperoxidase Assay}

Browning of acrosome, equatorial segment and whole head surface in the presence of blood serum or seminal

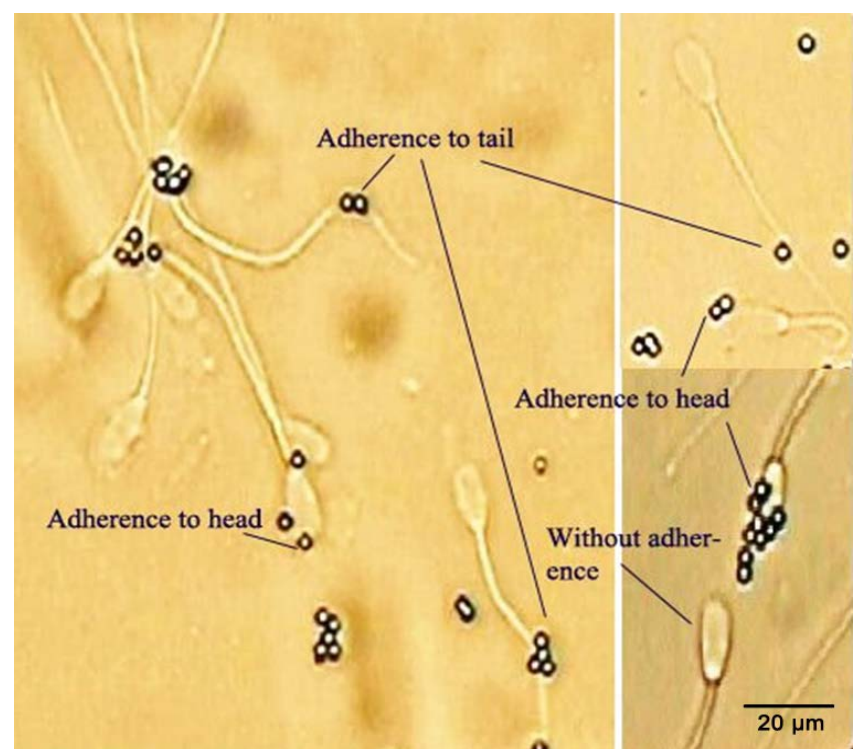

Figure 2. Showing adherence of latex particles of IgG/IgA class to various parts of cattle bull spermatozoa. 
plasma indicated the development of antibodies against surface proteins of acrosome, equatorial segment and whole head in bulls (Figure 1). There was 39.5\% $\pm 2.1 \%$ and $8.2 \% \pm 1 \%$ browning of acrosome, equatorial segment and whole head surface in the presence of blood serum and seminal plasma of bulls respectively. However, values ranged from $14.4 \%-57.8 \%$ and $0 \%-19.3 \%$ in blood serum and seminal plasma, respectively. It indicated the presence of $39.5 \% \pm 2.1 \%$ and $8.2 \% \pm 1 \%$ ASA in blood serum and seminal plasma against sperm head surface antigens, respectively (Table 1).

\subsection{Indirect Sperm Mar Test}

Binding of $\operatorname{IgA} / \operatorname{IgG}$ latex particles to sperm head, tail and head + tail indicated the development of antibodies of IgA/IgG type against sperm surface antigens in the blood serum or seminal plasma (Figure 2). Mean values for attachment of IgG type latex particles to motile spermatozoa were $41.2 \% \pm 2 \%$ and $2.4 \% \pm 0.4 \%$, ranging from $21.5 \%-62.5 \%$ and $0 \%-5.4 \%$ in the presence of blood serum and seminal plasma, respectively. Whereas, IgA latex particles attached to a mean of $26.4 \% \pm 1.7 \%$ and $10.7 \% \pm 1.5 \%$ motile spermatozoa, ranging from $7.3 \%-$ $39.8 \%$ and $0 \%-29.2 \%$ in the presence of blood serum and seminal plasma, respectively (Table 1 ).

\subsection{Enzyme-Linked Immunosorbent Assay}

Mean ELISA titre for ASA were $2361.5 \pm 258.2$ and 35.9 \pm 5.1 and ranged from $2000-6400$ and 0 - 80 in blood serum and seminal plasma of tested bulls (Table 1). Mean $28.5 \pm 1.0$ per cent positivity in the range of $18.5-$ 34.7 was detected in the blood serum of tested bulls, which was significantly higher $(\mathrm{P}<0.05)$ than that of negative control. It again indicated the presence of ASA in blood serum of breeding bulls.

Percentage of bulls with higher level of ASA, detected by IPA, Sperm Mar test and ELISA in serum and seminal plasma is given in Figure 3.

\subsection{Relationship between ASA in Blood Serum and Seminal Plasma of Bulls and Sperm Function/Fertility Tests}

\subsubsection{Immunoperoxidase Assay}

Sperm parameters of bulls with $>40$ vs. $<40 \%$ ASA in blood serum and $>20$ vs. $<20 \%$ ASA in seminal plasma were compared (Table 2$)$. A positive correlation was found between post-thawed motile $(+0.18)$, viabile $(+0.17)$, HOST $(+0.18)$, acrosome reacted $(+0.13)$ and cervical mucus penetrated spermatozoa in peak $0.5 \mathrm{~cm}(+0.19)$ and ASA in blood serum. HOS-positive and capacitated/acrosome reacted spermatozoa showed a significant

Table 1. Percent ASA (IPA), tested by IPA, SpermMar test and ELISA in serum and seminal plasma of cattle bulls.

\begin{tabular}{|c|c|c|c|c|c|c|c|c|}
\hline & \multirow{2}{*}{\multicolumn{2}{|c|}{ IPA $(\%)$}} & \multicolumn{4}{|c|}{ SpermMar test } & \multirow{2}{*}{\multicolumn{2}{|c|}{ ELISA (titre) }} \\
\hline & & & \multicolumn{2}{|c|}{$\operatorname{IgG}(\%)$} & \multicolumn{2}{|c|}{$\operatorname{IgA}(\%)$} & & \\
\hline & Serum & SP & Serum & SP & Serum & SP & Serum & SP \\
\hline Mean $\pm \mathrm{SE}$ & $39.5 \pm 2.1$ & $8.2 \pm 1.0$ & $41.2 \pm 2.0$ & $2.4 \pm 0.4$ & $26.4 \pm 1.7$ & $10.7 \pm 1.5$ & $2361.5 \pm 258.2$ & $35.9 \pm 5.1$ \\
\hline Range & $14.4-57.8$ & $0-19.3$ & $21.5-62.5$ & $0-5.4$ & $7.3-39.8$ & $0-29.2$ & $200-6400$ & $0-80$ \\
\hline
\end{tabular}

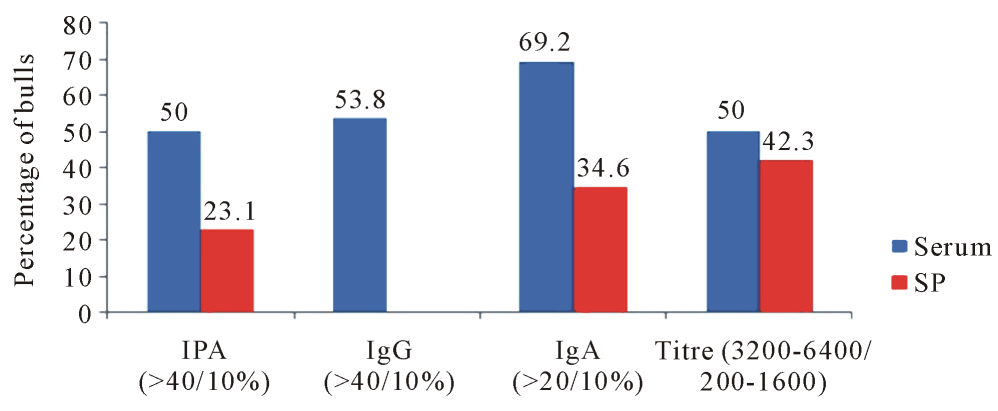

Figure 3. Occurance of ASA in total tested bulls. 
difference $(45.4 \% \pm 3.5 \% / 36.5 \% \pm 2.5 \%$ vs. $39.6 \% \pm 3.3 \% / 31.1 \% \pm 2.9 \%, \mathrm{P}<0.05)$ between the two groups (Table 2). Sperm count (million/ml) and percent HOS-positive spermatozoa were significantly higher $(\mathrm{P}<0.05$; $991.3 \pm 79.3$ vs. $879.5 \pm 37.3,43.1 \pm 2.9$ vs. $39.8 \pm 4.0)$ in bulls with $<20 \%$ seminal plasma ASA. Significant correlation $(\mathrm{P}<0.05)$ between seminal plasma ASA and post-thaw motile, distance covered by spermatozoa in cervical mucus $/ 30 \mathrm{~min}$ and number of spermatozoa penetrated in peak $0.5 \mathrm{~cm}$ was high $(+0.62)$, moderate $(+0.32)$ and weak $(+0.19)$, respectively.

\subsubsection{SpermMar Test (IgG)}

There was no difference in post-thaw motile, viable, acrosome intact/cervical mucus penetrated spermatozoa in the bulls with $>40 \%$ and $<40 \%$ blood serum IgG. Mean HOS-positive and acrosome reacted spermatozoa were higher $(47 \% \pm 3.4 \%$ Vs $37.1 \% \pm 2.8 \%$ and $35.5 \% \pm 2.1 \%$ Vs $30.4 \% \pm 2.9 \%)$ in bulls with $>40 \%$ IgG than with $<40 \%$ IgG (Table 3) and there was also significant correlation $(\mathrm{P}<0.05 ; \mathrm{r}=+0.22,+0.25)$ between the two. However, none of the tested bulls was with $>10 \%$ IgG in seminal plasma.

\subsubsection{Sperm Mar test (IgA)}

Abnormal/in vitro acrosome reacted and HOS-positive/acrosome intact spermatozoa were significantly $(\mathrm{P}<0.05)$

Table 2. Relationship between ASA (IPA) in serum and seminal plasma of bulls and sperm function/fertility tests.

\begin{tabular}{|c|c|c|c|c|c|c|c|c|c|}
\hline \multirow{2}{*}{$\begin{array}{l}\text { Percent ASA } \\
\text { (\% of bulls) }\end{array}$} & \multirow{2}{*}{$\begin{array}{l}\text { Post thaw } \\
\text { motility (\%) }\end{array}$} & \multirow{2}{*}{$\begin{array}{l}\text { Viability } \\
(\%)\end{array}$} & \multirow{2}{*}{$\begin{array}{c}\text { Abnormalities } \\
(\%)\end{array}$} & \multirow{2}{*}{$\begin{array}{l}\text { HOST } \\
(\%)\end{array}$} & \multirow{2}{*}{$\begin{array}{c}\text { Acrosome } \\
\text { reaction }(\%)\end{array}$} & \multicolumn{2}{|c|}{ CMPT } & \multirow{2}{*}{$\begin{array}{c}\text { Acrosome } \\
\text { integrity }(\%)\end{array}$} & \multirow{2}{*}{$\begin{array}{l}\text { Sperm count } \\
(\mathrm{ml} / \mathrm{million})\end{array}$} \\
\hline & & & & & & $\begin{array}{l}\text { Distance } \\
(\mathrm{mm})\end{array}$ & $\begin{array}{l}\text { Sperm } \\
\text { count }\end{array}$ & & \\
\hline \multicolumn{10}{|c|}{ Blood serum } \\
\hline$>40 \%(50)$ & $\begin{array}{c}41.2 \pm 0.8^{\mathrm{a}} \\
(35-45)\end{array}$ & $\begin{array}{c}65.4 \pm 2.3^{\mathrm{a}} \\
(55.2-84.8)\end{array}$ & $\begin{array}{l}16.9 \pm 1.2^{\mathrm{a}} \\
(9.7-23.2)\end{array}$ & $\begin{array}{l}45.4 \pm 3.5^{\mathrm{a}} \\
(31.1-66)\end{array}$ & $\begin{array}{c}36.5 \pm 2.5^{\mathrm{a}} \\
(26.5-41.4)\end{array}$ & $\begin{array}{c}22.9 \pm 2.0^{\mathrm{a}} \\
(12-28)\end{array}$ & $\begin{array}{c}363.7 \pm 29.7^{\mathrm{a}} \\
(231-578)\end{array}$ & $\begin{array}{c}88 \pm 3.6^{\mathrm{a}} \\
(61.3-100)\end{array}$ & $\begin{array}{r}898.9 \pm 46.2^{a} \\
(655-1178)\end{array}$ \\
\hline$<40 \%(50)$ & $\begin{array}{c}40.4 \pm 1.1^{\mathrm{a}} \\
(30-50)\end{array}$ & $\begin{array}{l}66.7 \pm 2.2^{\mathrm{a}} \\
(58-80.2)\end{array}$ & $\begin{array}{c}17.7 \pm 1.2^{\mathrm{a}} \\
(11.1-24.3)\end{array}$ & $\begin{array}{c}39.6 \pm 3.3^{\mathrm{b}} \\
(24.6-58.7)\end{array}$ & $\begin{array}{l}31.1 \pm 2.9^{b} \\
(13.1-50)\end{array}$ & $\begin{array}{c}23.0 \pm 2.5^{\mathrm{a}} \\
(9.0-37)\end{array}$ & $\begin{array}{c}375.5 \pm 30.3^{\mathrm{a}} \\
(219-529)\end{array}$ & $\begin{array}{c}87.6 \pm 3.6^{\mathrm{a}} \\
(66.2-98.3)\end{array}$ & $\begin{array}{l}882.2 \pm 48.4^{a} \\
(573-1301)\end{array}$ \\
\hline \multicolumn{10}{|c|}{ Seminal plasma } \\
\hline$>20 \%(23)$ & $\begin{array}{c}44.2 \pm 1.6^{\mathrm{a}} \\
(40-50)\end{array}$ & $\begin{array}{l}71.6 \pm 4.2^{\mathrm{a}} \\
(60-84.8)\end{array}$ & $\begin{array}{l}13.5 \pm 1.3^{\mathrm{a}} \\
(11.1-1.7)\end{array}$ & $\begin{array}{l}39.8 \pm 4.0^{\mathrm{a}} \\
(29.5-53)\end{array}$ & $\begin{array}{c}33.7 \pm 1.9^{\mathrm{a}} \\
(26.5-38.3)\end{array}$ & $\begin{array}{c}26.2 \pm 3.0^{\mathrm{a}} \\
(18-37)\end{array}$ & $\begin{array}{c}423.8 \pm 40.6^{\mathrm{a}} \\
(297-529)\end{array}$ & $\begin{array}{c}92.8 \pm 4.3^{\mathrm{a}} \\
(71.2-100)\end{array}$ & $\begin{array}{r}991.3 \pm 79.3^{a} \\
(744-1301)\end{array}$ \\
\hline
\end{tabular}

${ }^{\mathrm{a}}$ Figures in parentheses represent range; ${ }^{\mathrm{b}}$ Values with different superscripts are significant $(\mathrm{P}<0.5)$.

Table 3. Relationship between ASA (IgG type) in blood serum and seminal plasma of bulls and sperm function/fertility tests.

\begin{tabular}{|c|c|c|c|c|c|c|c|c|c|}
\hline \multirow{2}{*}{$\begin{array}{c}\text { Percent ASA } \\
\text { ( } \% \text { of bulls) }\end{array}$} & \multirow{2}{*}{$\begin{array}{l}\text { Post thaw } \\
\text { motility }(\%)\end{array}$} & \multirow{2}{*}{$\begin{array}{c}\text { Viability } \\
(\%)\end{array}$} & \multirow{2}{*}{$\begin{array}{c}\text { Abnormalities } \\
(\%)\end{array}$} & \multirow{2}{*}{$\begin{array}{c}\text { HOST } \\
(\%)\end{array}$} & \multirow{2}{*}{$\begin{array}{c}\text { Acrosome } \\
\text { reaction }(\%)\end{array}$} & \multicolumn{2}{|c|}{ CMPT } & \multirow{2}{*}{$\begin{array}{c}\text { Acrosome } \\
\text { integrity }(\%)\end{array}$} & \multirow{2}{*}{$\begin{array}{l}\text { Sperm count } \\
(\text { million } / \mathrm{ml})\end{array}$} \\
\hline & & & & & & $\begin{array}{l}\text { Distance } \\
(\mathrm{mm})\end{array}$ & $\begin{array}{l}\text { Sperm } \\
\text { count }\end{array}$ & & \\
\hline \multicolumn{10}{|c|}{ Blood seum } \\
\hline$>40 \%(53.7)$ & $\begin{array}{l}40 \pm 1.0^{\mathrm{a}} \\
(30-45)\end{array}$ & $\begin{array}{c}66.1 \pm 2.4^{\mathrm{a}} \\
(51.9-84.8)\end{array}$ & $\begin{array}{l}16.2 \pm 1.1^{\mathrm{a}} \\
(9.7-23.2)\end{array}$ & $\begin{array}{c}47 \pm 3.4^{\mathrm{a}} \\
(28.7-58.7)\end{array}$ & $\begin{array}{c}35.5 \pm 2.1^{\mathrm{a}} \\
(26.5-43.1)\end{array}$ & $\begin{array}{l}23.1 \pm 1.7^{\mathrm{a}} \\
(1.2-3.3)\end{array}$ & $\begin{array}{c}385.3 \pm 29.5^{a} \\
(231-578)\end{array}$ & $\begin{array}{c}88.6 \pm 3.5^{\mathrm{a}} \\
(61.3-100)\end{array}$ & $\begin{array}{c}900.8 \pm 46.9^{a} \\
(573-1178)\end{array}$ \\
\hline$<40 \%(46.3)$ & $\begin{array}{c}41.6 \pm 0.9^{\mathrm{a}} \\
(40-50)\end{array}$ & $\begin{array}{c}65.9 \pm 2.3^{\mathrm{a}} \\
(57.3-80.2)\end{array}$ & $\begin{array}{c}18.2 \pm 1.2^{\mathrm{a}} \\
(11.3-23.2)\end{array}$ & $\begin{array}{c}37.1 \pm 2.8^{b} \\
(24.6-58.6)\end{array}$ & $\begin{array}{l}30.4 \pm 2.9^{\mathrm{a}} \\
(13.1-50)\end{array}$ & $\begin{array}{c}22.3 \pm 2.6^{\mathrm{a}} \\
(9.0-37)\end{array}$ & $\begin{array}{c}381.2 \pm 32.3^{\mathrm{a}} \\
(219-529)\end{array}$ & $\begin{array}{c}88 \pm 3.4^{\mathrm{a}} \\
(66.2-98.3)\end{array}$ & $\begin{array}{c}910.5 \pm 50.5^{\mathrm{a}} \\
(655-1301)\end{array}$ \\
\hline
\end{tabular}

Seminal plasma

\begin{tabular}{|c|c|c|c|c|c|c|c|c|c|}
\hline$<10 \%(100)$ & $\begin{array}{c}40.7 \pm 0.5^{\mathrm{a}} \\
(30-50)\end{array}$ & $\begin{array}{c}66 \pm 1.7^{\mathrm{a}} \\
(51.9-84.8)\end{array}$ & $\begin{array}{c}17 \pm 0.8^{\mathrm{a}} \\
(9.7-24.3)\end{array}$ & $\begin{array}{l}42.4 \pm 2.4^{\mathrm{a}} \\
(24.6-66)\end{array}$ & $\begin{array}{c}33.1 \pm 1.7^{\mathrm{a}} \\
(13.1-54.9)\end{array}$ & $\begin{array}{c}23.1 \pm 0.2^{\mathrm{a}} \\
(9.0-37)\end{array}$ & $\begin{array}{c}383.3 \pm 21.3^{\mathrm{a}} \\
(219-578)\end{array}$ & $\begin{array}{c}87.1 \pm 2.6^{\mathrm{a}} \\
(61.3-100)\end{array}$ & $\begin{array}{c}870.6 \pm 46.6^{a} \\
(573-1301)\end{array}$ \\
\hline
\end{tabular}

${ }^{\mathrm{a}}$ Figures in parentheses represent range; ${ }^{\mathrm{b}}$ Values with different superscripts are significant $(\mathrm{P}<0.05)$. 
and non-significantly $(\mathrm{P}>0.05)$ higher in bulls with $>20 \%$ than $<20 \%$ serum IgA, respectively (Table 4). HOSpositive, in vitro acrosome reacted and acrosome intact spermatozoa were non-significantly $(\mathrm{P}>0.05)$ higher in bulls with $<10 \%$ than with $>10 \%$ seminal plasma IgA. Whereas, number of spermatozoa in peak $0.5 \mathrm{~cm}$ in cervical mucus was significantly $(\mathrm{P}<0.05)$ higher in bulls with $<10 \%$ seminal plasma $\operatorname{IgA}$ than $>10 \%$ and there was also a significant correlation $(\mathrm{r}=+0.36)$ between seminal plasma IgA and CMPT. Sperm count ( $\mathrm{million} / \mathrm{ml})$ in ejaculated semen was also significantly $(\mathrm{P}<0.05)$ different among the two groups.

\subsubsection{Enzyme-Linked Immunosorbent Assay}

There was not much difference in motile, viable, abnormal morphology, HOS-positive, acrosome intact, in vitro arosome reacted and cervical mucus penetrated spermatozoa between the bulls with $3200-6400$ and $<3200$ serum antibody titre (Table 5). But a very weak correlation $(+0.004$ to +0.17$)$ was found between serum ELISA titre and abnormal, viable, motile, HOS-positive, in vitro arosome reacted and cervical mucus penetrated spermatozoa. Acrosome intact, sperm count (million/ml) and numbers of spermatozoa penetrated in peak $0.5 \mathrm{~cm}$ of cervical mucus were higher in bulls with seminal plasma antibody titre of $0-20$ than that of $40-80$, but difference was significant only in later two parameters. Seminal plasma antibody titre and motile/cervical mucus penetrated spermatozoa also showed a moderate positive correlation ( +0.49 and $+0.28 /+0.37$ ).

Table 4. Relationship between ASA (IgA type) in blood serum and seminal plasma of bulls and sperm function/fertility tests.

\begin{tabular}{|c|c|c|c|c|c|c|c|c|c|}
\hline \multirow{2}{*}{$\begin{array}{c}\text { Percent ASA } \\
\text { ( } \% \text { of bulls) }\end{array}$} & \multirow{2}{*}{$\begin{array}{l}\text { Post thaw } \\
\text { motility (\%) }\end{array}$} & \multirow{2}{*}{$\begin{array}{l}\text { Viability } \\
(\%)\end{array}$} & \multirow{2}{*}{$\begin{array}{c}\text { Abnormalities } \\
(\%)\end{array}$} & \multirow{2}{*}{$\begin{array}{l}\text { HOST } \\
(\%)\end{array}$} & \multirow{2}{*}{$\begin{array}{l}\text { Acrosome } \\
\text { reaction (\%) }\end{array}$} & \multicolumn{2}{|c|}{ CMPT } & \multirow{2}{*}{$\begin{array}{l}\text { Acrosome } \\
\text { integrity (\%) }\end{array}$} & \multirow{2}{*}{$\begin{array}{l}\text { Sperm count } \\
(\text { million } / \mathrm{ml})\end{array}$} \\
\hline & & & & & & $\begin{array}{l}\text { Distance } \\
(\mathrm{mm})\end{array}$ & $\begin{array}{l}\text { Sperm } \\
\text { count }\end{array}$ & & \\
\hline \multicolumn{10}{|c|}{ Blood serum } \\
\hline$>20 \%(69.2)$ & $\begin{array}{c}40.5 \pm 0.9^{\mathrm{a}} \\
(30-45)\end{array}$ & $\begin{array}{c}66.9 \pm 2.2^{\mathrm{a}} \\
(51.9-84.8)\end{array}$ & $\begin{array}{l}15.7 \pm 0.9^{\mathrm{a}} \\
(9.7-23.2)\end{array}$ & $\begin{array}{c}45 \pm 2.9^{\mathrm{a}} \\
(28.7-54.2)\end{array}$ & $\begin{array}{c}34.8 \pm 1.7^{\mathrm{a}} \\
(26.2-54.9)\end{array}$ & $\begin{array}{l}24 \pm 1.7^{\mathrm{a}} \\
(9.0-37)\end{array}$ & $\begin{array}{c}383.7 \pm 24.9^{\mathrm{a}} \\
(231-578)\end{array}$ & $\begin{array}{c}90.1 \pm 3.0^{\mathrm{a}} \\
(61.3-100)\end{array}$ & $\begin{array}{r}908.9 \pm 39.6^{\mathrm{a}} \\
(573-1178)\end{array}$ \\
\hline$<20 \%(30.8)$ & $\begin{array}{c}41.2 \pm 1.2^{\mathrm{a}} \\
(40-50)\end{array}$ & $\begin{array}{l}64.1 \pm 2.0^{\mathrm{a}} \\
(58-74.4)\end{array}$ & $\begin{array}{c}20.1 \pm 1.3^{b} \\
(14.5-23.2)\end{array}$ & $\begin{array}{c}36.6 \pm 3.8^{\mathrm{a}} \\
(24.6-58.6)\end{array}$ & $\begin{array}{l}20.1 \pm 1.3^{b} \\
(13.15-50)\end{array}$ & $\begin{array}{c}22.1 \pm 3.0^{\mathrm{a}} \\
(11-35)\end{array}$ & $\begin{array}{c}382.5 \pm 43.3^{\mathrm{a}} \\
(219-528)\end{array}$ & $\begin{array}{c}86.7 \pm 4.1^{\mathrm{a}} \\
(66.2-96.6)\end{array}$ & $\begin{array}{l}897.1 \pm 72.4^{\mathrm{a}} \\
(655-1301)\end{array}$ \\
\hline \multicolumn{10}{|c|}{ Seminal plasma } \\
\hline$>10 \%(34.6)$ & $\begin{array}{c}42.8 \pm 1.2^{\mathrm{a}} \\
(40-50)\end{array}$ & $\begin{array}{c}68.9 \pm 3.5^{\mathrm{a}} \\
(56.6-84.8)\end{array}$ & $\begin{array}{c}14.6 \pm 1.1^{\mathrm{a}} \\
(11.1-20.6)\end{array}$ & $\begin{array}{l}37.9 \pm 2.9^{\mathrm{a}} \\
(29.5-53)\end{array}$ & $\begin{array}{c}31.6 \pm 1.7^{\mathrm{a}} \\
(23.3-38.3)\end{array}$ & $\begin{array}{l}24 \pm 3.2^{\mathrm{a}} \\
(9.0-37)\end{array}$ & $\begin{array}{l}427 \pm 33.6^{\mathrm{a}} \\
(297-578)\end{array}$ & $\begin{array}{c}86.9 \pm 4.6^{\mathrm{a}} \\
(66.2-100)\end{array}$ & $\begin{array}{r}971.5 \pm 60.5^{\mathrm{a}} \\
(744-1301)\end{array}$ \\
\hline$<10 \%(65.4)$ & $\begin{array}{c}39.8 \pm 0.7^{\mathrm{a}} \\
(30-45)\end{array}$ & $\begin{array}{c}64.6 \pm 1.5^{\mathrm{a}} \\
(51.9-71.8)\end{array}$ & $\begin{array}{l}18.3 \pm 1.0^{\mathrm{a}} \\
(9.7-24.3)\end{array}$ & $\begin{array}{l}44.8 \pm 3.4^{\mathrm{a}} \\
(24.6-66)\end{array}$ & $\begin{array}{c}34 \pm 2.5^{\mathrm{a}} \\
(13.15-54.9)\end{array}$ & $\begin{array}{c}22.1 \pm 1.5^{\mathrm{a}} \\
(11-31)\end{array}$ & $\begin{array}{l}460 \pm 26.4^{\mathrm{b}} \\
(219-528)\end{array}$ & $\begin{array}{l}89.2 \pm 2.8^{\mathrm{a}} \\
(61.3-7.2)\end{array}$ & $\begin{array}{c}870.2 \pm 39.5^{\mathrm{b}} \\
(573-1178)\end{array}$ \\
\hline
\end{tabular}

${ }^{\mathrm{a}}$ Figures in parentheses represent range; ${ }^{\mathrm{b}}$ Values with different superscripts are significant $(\mathrm{P}<0.05)$.

Table 5. Relationship between ASA (ELISA) in blood serum and seminal plasma of bulls and sperm function/fertility tests.

\begin{tabular}{|c|c|c|c|c|c|c|c|c|c|}
\hline \multirow[b]{2}{*}{$\begin{array}{c}\text { Titre } \\
\text { (\% of bulls) }\end{array}$} & \multirow[b]{2}{*}{$\begin{array}{l}\text { Post thaw } \\
\text { motility (\%) }\end{array}$} & \multirow[b]{2}{*}{$\begin{array}{c}\text { Viability } \\
(\%)\end{array}$} & \multirow[b]{2}{*}{$\begin{array}{c}\text { Abnormalities } \\
(\%)\end{array}$} & \multirow[b]{2}{*}{$\begin{array}{l}\text { HOST } \\
(\%)\end{array}$} & \multirow[b]{2}{*}{$\begin{array}{l}\text { Acrosome } \\
\text { reaction (\%) }\end{array}$} & \multicolumn{2}{|c|}{ CMPT } & \multirow[b]{2}{*}{$\begin{array}{l}\text { Acrosome } \\
\text { integrity (\%) }\end{array}$} & \multirow[b]{2}{*}{$\begin{array}{l}\text { Sperm count } \\
\text { (million } / \mathrm{ml} \text { ) }\end{array}$} \\
\hline & & & & & & $\begin{array}{c}\text { Distance } \\
(\mathrm{mm})\end{array}$ & $\begin{array}{l}\text { Sperm } \\
\text { count }\end{array}$ & & \\
\hline
\end{tabular}

\begin{tabular}{|c|c|c|c|c|c|c|c|c|c|}
\hline \multicolumn{10}{|c|}{ Blood serum } \\
\hline $\begin{array}{c}200-1600 \\
(50)\end{array}$ & $\begin{array}{c}40.8 \pm 1.3^{\mathrm{a}} \\
(35-45)\end{array}$ & $\begin{array}{c}67.1 \pm 2.2^{\mathrm{a}} \\
(51.9-84.8)\end{array}$ & $\begin{array}{l}17.7 \pm 1.3^{\mathrm{a}} \\
(9.7-23.2)\end{array}$ & $\begin{array}{l}40.5 \pm 3.6^{\mathrm{a}} \\
(28.7-66)\end{array}$ & $\begin{array}{c}32.9 \pm 2.5^{\mathrm{a}} \\
(13.1-54.9)\end{array}$ & $\begin{array}{l}2.3 \pm 2.3^{\mathrm{a}} \\
(9.0-33)\end{array}$ & $\begin{array}{l}380 \pm 31.9^{a} \\
(231-578)\end{array}$ & $\begin{array}{c}86.9 \pm 3.5^{a} \\
(61.3-100)\end{array}$ & $\begin{array}{c}917 \pm 48.9^{a} \\
(655-1301)\end{array}$ \\
\hline $\begin{array}{c}3200-6400 \\
(50)\end{array}$ & $\begin{array}{c}40.7 \pm 0.7^{\mathrm{a}} \\
(30-50)\end{array}$ & $\begin{array}{c}65.1 \pm 24^{\mathrm{a}} \\
(57.3-80.2)\end{array}$ & $\begin{array}{c}16.6 \pm 1.1^{\mathrm{a}} \\
(11.1-24.3)\end{array}$ & $\begin{array}{c}44.1 \pm 3.4^{\mathrm{a}} \\
(24.6-58.7)\end{array}$ & $\begin{array}{l}33.2 \pm 2.5^{\mathrm{a}} \\
(16.1-50)\end{array}$ & $\begin{array}{l}2.2 \pm 2.0^{\mathrm{a}} \\
(11-37)\end{array}$ & $\begin{array}{l}385 \pm 29.7^{\mathrm{a}} \\
(219-529)\end{array}$ & $\begin{array}{c}89.8 \pm 3.0^{\mathrm{a}} \\
(66.3-98.3)\end{array}$ & $\begin{array}{c}894.5 \pm 47.8^{a} \\
(573-1178)\end{array}$ \\
\hline \multicolumn{10}{|c|}{ Seminal plasma } \\
\hline $\begin{array}{l}0-20 \\
(57.7)\end{array}$ & $\begin{array}{c}41.8 \pm 1.0^{\mathrm{a}} \\
(40-50)\end{array}$ & $\begin{array}{c}66.8 \pm 2.77^{\mathrm{a}} \\
(56.6-84.8)\end{array}$ & $\begin{array}{c}15.7 \pm 1.3^{\mathrm{a}} \\
(11.5-24.3)\end{array}$ & $\begin{array}{l}39.7 \pm 3.7^{\mathrm{a}} \\
(28.7-66)\end{array}$ & $\begin{array}{c}32.6 \pm 1.8^{\mathrm{a}} \\
(23.3-43.1)\end{array}$ & $\begin{array}{c}22.9 \pm 0.8^{\mathrm{a}} \\
(9.0-37)\end{array}$ & $\begin{array}{l}447 \pm 27.4^{a} \\
(297-578)\end{array}$ & $\begin{array}{c}84.8 \pm 4.3^{\mathrm{a}} \\
(61.3-100)\end{array}$ & $\begin{array}{l}863.1 \pm 42.1^{\mathrm{a}} \\
(573-1178)\end{array}$ \\
\hline
\end{tabular}

${ }^{a}$ Figures in parentheses represent range; ${ }^{b}$ Values with different superscripts are significant $(\mathrm{P}<0.05)$. 


\section{Discussion}

\subsection{Anti Sperm Antibodies}

SpermMar test indicated the presence of very high percentage of IgG in blood serum $(41.2 \% \pm 2 \%)$ as compared with seminal plasma $(2.4 \% \pm 0.4 \%)$, but $\operatorname{IgA}(10.7 \% \pm 1.5 \%)$ class ASA was in higher percentage than $\operatorname{IgG}(2.4 \%$ $\pm 0.4 \%$ ) in seminal plasma. Higher percentage of $\operatorname{IgA}$ in seminal plasma may be due to the reason that these are secreted by the accessory sex glands [14]. IgA class antibodies are present in seminal plasma and also attach to the sperm surface, but are usually absent in serum. Milovanovic et al. [15] confirmed the hypothesis that immune mechanisms might be involved in reproductive disturbances due to high levels of ASA of IgA class. ASA of the IgA class, which mainly has agglutinating properties [16], rarely occurs without antibodies of the IgG class. Therefore, both classes are important for male infertility.

IPA, SpermMar test and ELISA confirmed the occurrence of ASA in blood serum and seminal plasma of 26 and 21 tested bulls, respectively. It has been suggested that molecular mimicry between bacteria and the sperm can be a major factor inducing antisperm immunological reactions [17]. In SpermMar test, 40\% reaction between motile spermatozoa and coated latex particles of IgG class is considered as the lower limit of significant activity. In general, the proportion of motile spermatozoa reacting in the SpermMar-IgA test is smaller than that reacting in the SpermMar-IgG test, but the contrary may occasionally occur [18]. In rare cases, there is a positive reaction in the SpermMar-IgA test in the absence of any reaction in the SpermMar-IgG test, indicating the presence of antibodies of the IgA class without antibodies of the IgG class. Therefore, bulls with $>40 \%$ IPA/ IgG, $>20 \%$ IgA, $3200-6400$ titre and $>0 \%$ IPA/IgG/IgA, $40-80$ ELISA titre in blood serum and seminal plasma, respectively were considered for significant presence of ASA. In SpermMar test, about 54\% bulls were with $>40 \%$ IgG in blood serum against sperm surface antigens, but none of the bulls were with $>10 \% \operatorname{IgG}$ in seminal plasma. Higher percentage of IgA against sperm surface antigens was detected in the blood serum $(>20 \%)$ and seminal plasma $(>10 \%)$ of $65.8 \%$ and $37 \%$ bulls, respectively. In IPA, about $50 \%$ of the bulls also had $>40 \%$ ASA against head surface antigens, whereas, there were only $23 \%$ bulls with $>10 \%$ ASA in seminal plasma. ELISA indicated a higher antibody titre in blood serum (3200 - 6400) and seminal plasma (40 - 80) of 50\% and $42 \%$ bulls, respectively. Out of 26 tested bulls, seminal plasma of 21 bulls reacted with spermatozoa both in IPA and IgA latex particles and that of 12 bulls reacted only with IgG. This indicated the presence of IgA class antibodies without $\mathrm{IgG}$ in seminal plasma of $57.1 \%$ tested bulls. Occurrence of mixed agglutination reaction of $40 \%$ or more in semen indicates a positive reaction to the SpermMar-IgA test. Although mixed reaction of IgG and IgA was not $>40 \%$ in seminal plasma of all tested bulls, IgG class antibody was $>40 \%$ in serum of $54 \%$ of tested bulls. Therefore, a combination of tests revealed higher percentage of ASA in blood serum of about $50 \%$ of the tested bulls. Higher level of ASA in serum than seminal plasma indicated that ASA in blood serum of bulls was of circulatory type. IgG/IgA class antibodies in the blood can cross testis/epididymis and may affect spermatogenesis and sperm maturation; therefore, elevated level of ASA in blood serum of $50 \%$ tested bulls may have effects on the process of sperm maturation in these bulls.

\subsection{Relationship between ASA in Blood Serum and Seminal Plasma of Bulls and Sperm Function/Fertility Tests}

Higher mean values of motile, viable, intact acrosomes, capacitated/acrosome reacted spermatozoa in bulls with $>20 \%$ serum-ASA (IPA) as compared to $<20 \%$ ASA observed during the present study may be due to individual variation. Romano et al. [19] also found that the proportion of acrosome reacted spermatozoa was higher in ASA-coated spermatozoa. The immunological defense is activated and production of ASA initiated, when there is both acute and chronic infection and/or inflammation, especially of the epididymis [20] [21]. First, IgM antibodies will be produced, but these are not secreted into the genital tract because their size is too large to pass the epithelial barrier. Shortly afterwards, antibodies of the IgG class appear, and these can enter the genital tract. The ASA of the IgG class come into contact and attach with the spermatozoa [22]. During the present study, inspite of higher percentage of $(>40 \%)$ IgG in serum of $53.7 \%$ bulls, sperm parametes were not affected. It is possible that IgG against spermatozoa were present in serum of these bulls, but did not enter the genital tract.

Sperm parameters, i.e. HOST, acrosome integrity, in vitro acrosome reaction and CMPT were higher in bulls with $<10 \%$ IgA as compared to those with $>10 \%$ IgA in seminal plasma. In some cases and more commonly indeed during infection, secretory IgA-ASA are produced locally in the genital tract, probably the epididymis [23]. 
Antibodies of the IgA class can then be detected on the ejaculated spermatozoa, but not in serum, and this is associated with an additional reduction of their fertilizing capacity [14]. In the present study, IgA class antibodies of significance $(>10 \%)$ were produced in genital tract of only $57.1 \%$ bulls, which are attached to the spermatozoa and had effect on penetration of spermatozoa through cervical mucus and in vitro acrosome reacton. Milovanovic et al. [15] also gave the hypothesis that immune mechanism may be involved in reproductive disturbances due to high level of ASA of IgA class. Recently, a study done by Jarora et al. [24] indicated that higher percentage of IgG-ASA and IgA-ASA in cervical mucus of cross bred cows reduced in vitro penetration of spermatozoa through cervical mucus in $44 \%$ of the tested animals.

It can be concluded that higher level of ASA, especially IgA class antibodies in seminal plasma reduced post-thaw motility, in vitro capacitation/acrosome reaction and cervical mucus penetration of spermatozoa. Presence of ASA can inhibit passage of spermatozoa through cervical mucus, prevent membrane fluidity changes needed for capacitation, reduce the ability of spermatozoa to undergo the acrosome reaction, and interfere with binding to the zona pellucida and fertilization [25]. Experimentally induced ASA were shown to affect the ability of bull spermatozoa to fertilize oocytes in vitro [4], while naturally occurring ASA were associated with reduced spermatozoal motility and infertility in two bulls [26]. Various parts of the sperm are surrounded by a common plasma membrane. ASA have been shown to react with plasma membrane with variable biological effects [27].

There were only 5 bulls among those with $>40 \%$ blood serum ASA (IPA/IgG) and 3200 - 6400 antibody titre, who also had low values for HOST, in vitro acrosome reaction and CMPT. Among the bulls with $>10 \%$ seminal plasma IPA/IgA and 40 - 80 antibody titre, only 3 bulls had low values for HOS-positive, in vitro acrosome reacted and cervical mucus penetrated spermatozoa. There were also one and two bulls with higher level of seminal plasma ASA and low values of only CMPT/HOST and in vitro acrosome reaction, respectively. Therefore, there were 11 bulls with low values of HOST/in vitro acrosome reaction/CMPT and higher significant level of either serum - or seminal plasma-ASA. Hence, it can be interpreted that significanct level of serum/seminal plasma ASA may have effect on the fertility of bulls by affecting any of the sperm function, i.e. membrane integrity/ capacitation/acrosome reaction/CMPT. Moreover, in the absence of any standard and universally accepted assay for the detection of ASA, hence, as per our findings, it is suggested that combination of tests gives higher accuracy in comparison to any particular single test.

\section{References}

[1] Zraly, Z., Bendova, J., Diblikova, I., Svecova, D., Kummer, V., Maskova, J. and Veznik, Z. (2002) Antisperm Antibodies in Blood Sera of Bulls and Correlations with Age, Breed and Ejaculate Quality. Acta Veterinaria, 71, 303-308. http://dx.doi.org/10.2754/avb200271030303

[2] Murdoch, A.J., Buckley, C.H. and Fox, H. (1982) Hormonal Control of the Secretory Immune System of the Human Uterine Cervix. Journal of Reproductive Immunology, 4, 23-30. http://dx.doi.org/10.1016/0165-0378(82)90020-1

[3] Bronson, R.A., Cooper, G.W., Rosenfeld, D.L. and Witkin, S.S. (1984) Detection of Spontaneously Occurring SpermDirected Antibodies in Infertile Couples by Immunobead Binding and Enzyme-Linked Immunosorbent Assay. Annals of New York Academy of Sciences, 438, 504-507. http://dx.doi.org/10.1111/j.1749-6632.1984.tb38318.x

[4] Kim, C.A., Parrish, J.J., Momont, H.W. and Lunn, D.P. (1999) Effects of Experimentally Generated Bull Antisperm Antibodies on in Vitro Fertilization. Biology of Reproduction, 60, 1285-1291. http://dx.doi.org/10.1095/biolreprod60.6.1285

[5] Lombardo, F., Gandini, L., Dondero, F. and Lenzi, A. (2001) Immunology and Immunopathology of the Male Genital Tract: Antisperm Immunity in Natural and Assisted Reproduction. Human Reproduction Update, 7, 450-456. http://dx.doi.org/10.1093/humupd/7.5.450

[6] Blom, E. (1950) A One-Minute Live-Dead Sperm Stain by Means of Eosin-Nigrosin. Fertility Sterility, 1, 176-177.

[7] Correa, J.R. and Zavos, P.M. (1994) The Hypo-Osmotic Swelling Test: Its Employment as an Assay to Evaluate the Functional Integrity of the Frozen-Thawed Bovine Sperm Membrane. Theriogenology, 42, 351-360. http://dx.doi.org/10.1016/0093-691X(94)90280-1

[8] Murase, T. and Braun, J.W. (1990) Impact of Methodological Factors on Sperm Penetration into Cervical Mucus in Cattle. Theriogenology, 34, 73-80. http://dx.doi.org/10.1016/0093-691X(90)90578-H

[9] Sarma, D.K., Baishya, N., Sharma, D.K., Deka, B.C. and Bhuyan, D. (2009) Antisperm Antibodies in Serum and Cervical Mucus of Normal and Repeat Breeding Cows. Indian Journal of Animal Reproduction, 30, 54-56.

[10] Parrish, J.J., Susko-Parrish, J.L. and First, N.L. (1989) Capacitation of Bovine Sperm by Heparin: Inhibitory Effect of 
Glucose and Role of Intracellular pH. Biology of Reproduction, 41, 683-699. http://dx.doi.org/10.1095/biolreprod41.4.683

[11] Fayemi, O. (2005) Sperm Antibodies and Reproductive Efficiency in the Zebu Cattle in South Western Nigeria. Pakistan Veterinary Journal, 25, 111-114.

[12] FertiPro, N.V. (2011) A Qualitative Latex Test for Detection of Sperm Antibodies. Bijsluiter Sperm Mar Indd, 12, 31 32.

[13] Crowther, J.R. (1995) Methods in Molecular Biology. In: Crowther, J.R., Ed., ELISA: Theory and Practice, Humana Press, Totowa, 415-420.

[14] Comhaire, F.H., Mahmoud, A.M., Depuydt, C.E., Zalata, A.A. and Christophe, A.B. (1999) Mechanisms and Effects of Male Genital Tract Infection on Sperm Quality and Fertilizing Potential: The Andrologist's Viewpoint. Human Reproduction Update, 5, 393-398. http://dx.doi.org/10.1093/humupd/5.5.393

[15] Milovanovic, A., Lazarevic, M., Milanovic, S., Kirovski, D. and Jovicin, M. (2005) Open Days Period and Antispermatozoal Antibodies in Artificially Inseminated Cows. Acta Veterinaria, 55, 449-460. http://dx.doi.org/10.2298/AVB0506449M

[16] Stedronska, J. and Hendry, W.F. (1983) The Value of the Mixed Antiglobulin Reaction (MAR-Test) as an Addition to Routine Seminal Analysis in the Evaluation of the Subfertile Couple. American Journal of Reproduction, Immunology and Microbiology, 3, 89-91. http://dx.doi.org/10.1111/j.1600-0897.1983.tb00221.x

[17] Kurpisz, M. and Alexander, N.J. (1995) Carbohydrate Moieties on Sperm Surface: Physiological Relevance. Fertility and Sterility, 63, 158-165.

[18] Ackerman, S., McGuire, G., Fulgham, D.L. and Alexander, N. (1988) An Evaluation of a Commercially Available Assay for the Detection of Antisperm Antibodies. Fertility and Sterility, 49, 732-734.

[19] Romano, R., Santucci, R., Marrone, V. and Francavilla, F. (1993) Effect of Ionophore Challenge on Hamster Egg Penetration and Acrosome Reaction of Antibody-Coated Human Sperm. American Journal of Reproduction Immunology and Microbiology, 29, 56-61. http://dx.doi.org/10.1111/j.1600-0897.1993.tb00838.x

[20] Munoz, M.G. and Witkin, S.S. (1995) Autoimmunity to Spermatozoa, Asymptomatic Chlamydia trachomatis Genital Tract Infection and Gamma Delta T Lymphocytes in Seminal Fluid from the Male Partners of Couples with Unexplained Infertility. Human Reproduction Update, 10, 1070-1074.

[21] Witkin, S.S., Kligman, I. and Bongiovanni, A.M. (1995) Relationship between an Asymptomatic Male Genital Tract Exposure to Chlamydia trachomatis and an Autoimmune Response to Spermatozoa. Human Reproduction Update, 10, 2952-2955.

[22] Nikolaeva, M.A., Kulakov, V.I., Ter Avanesov, G.V., Terekhina, L.N., Pschenichinikova, T.J. and Sukhikh, G.T. (1993) Detection of Antisperm Antibodies on the Surface of Living Spermatozoa Using Flow Cytometry: Preliminary Study. Fertility and Sterility, 59, 639-644.

[23] Meinertz, H., Linnet, L., Wolf, H. and Hjort, T. (1991) Antisperm Antibodies on Epididymal Spermatozoa. American Journal of Reproductive Immunology, 25, 158-162. http://dx.doi.org/10.1111/j.1600-0897.1991.tb01087.x

[24] Jarora, V., Gandotra, V.K., Cheema, R.S., Bansal, A.K. and Dhindsa, S.S. (2014) Relationship of in Vitro Cervical Mucus Penetration Assay with IgA and IgG Type Antibodies in Cross-Bred Cows. Advances in Animal and Veterinary Sciences, 2, 606-611. http://dx.doi.org/10.14737/journal.aavs/2014/2.11.606.611

[25] Fijak, M. and Meinhardt, A. (2006) The Testis in Immune Privilege. Immunological Reviews, 213, 66-81. http://dx.doi.org/10.1111/j.1600-065X.2006.00438.x

[26] Hunter, A.G. (1989) Immunology and Fertility in the Bovine. Journal of Dairy Science, 72, 3353-3362. http://dx.doi.org/10.3168/jds.S0022-0302(89)79498-4

[27] Chamley, L.W. and Clarke, G.N. (2007) Antisperm Antibodies and Conception. Seminars in Immunopathology, 29, 169-184. http://dx.doi.org/10.1007/s00281-007-0075-2 